\title{
2019 WSGC Elijah High-Altitude Balloon Payload
}

\author{
Chance Beaty ${ }^{1}$, Eileen Endres ${ }^{2}$, Nicholas Hennigan ${ }^{2}$, Daniel Schuler ${ }^{2}$, \\ D'AmoureWashburn'2, and Katherine Wolfgramm² \\ ${ }^{1}$ Carthage College, ${ }^{2}$ Milwaukee School of \\ Engineering
}

\begin{abstract}
The 2019 WSGC Elijah High-Altitude Balloon Payload Fellowship focused on 4 high-altitude phenomena: Modular Payload Design, Applications of Air Turbulence (power generation and visualization), Electronic Behavior at Altitude, and Sonification of Atmospheric Data. Modular payload design focused on user-accessibility by creating friction reducing rings in-between insulation and the instrumentation capsule. Height-adjustable, modular shelving was also constructed. Turbulence and Electronics project both suffered data loss during flight. However, post-flight lab analysis showed power generation apparatus produced $96 \mathrm{~J}-120 \mathrm{~J}$ and turbulence visualization's potential to assist in calculating Eddie dissipation rates. Additionally, Electronic Behavior observed corona discharges across large electrical gaps near vacuum pressures. Sonification of Data used computer algorithms to transcribe data relationships into music. The intent was to allow users to perceive data relationships and patterns aurally.
\end{abstract}

\section{Overview}

The Elijah Balloon fellowship is a research program established by the consortium with the intention of enabling undergraduates in STEM fields to gain design research experience via a highaltitude balloon platform. This year's team explored different facets of high-altitude phenomena and as well as novel approaches to analyzing data. Research topics included: modular payload design concepts, ionization of air, power generation from flight turbulence, and turbulence visualization. Additionally, the research team looked at the sonification of data and how computer algorithms can help redefine the way humans perceive data. Instead of traditional graphical representations, the team explored how musical relationships can describe trends in data.

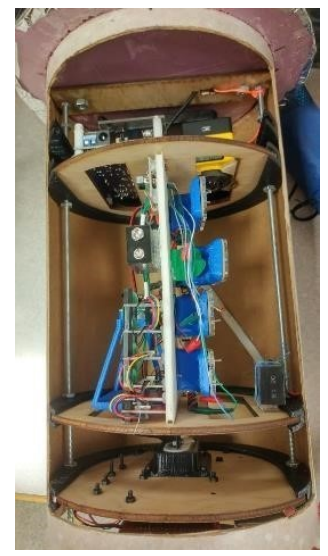

\subsection{Payload Design}

Design parameters for the payload focused around flexibility, ease of use and video capture. Individual bays and modular shelving were constructed to provide quick access to experiments on the field, as shown in Figure 1. Additionally, 3D printed guide rings were fabricated to reduce sliding friction between the outer protective housing and foam/mylar insulating composite shell (Figure 2). Prior years designs struggled with separating the insulation shell and experimental housing, this outer ring design was an attempt to address prior years difficulties. Another design objective was to construct a system in which the rocket tube did not slide with respect to the shell during flight. Any motion intercepted by the IMU's would be purely the result of the Figure 1. Payload Shelving motion of the payload eliminating relative velocities. A "snap and lock" model (Figure 2) was fabricated to ensure the elimination of relative velocities. The right image 

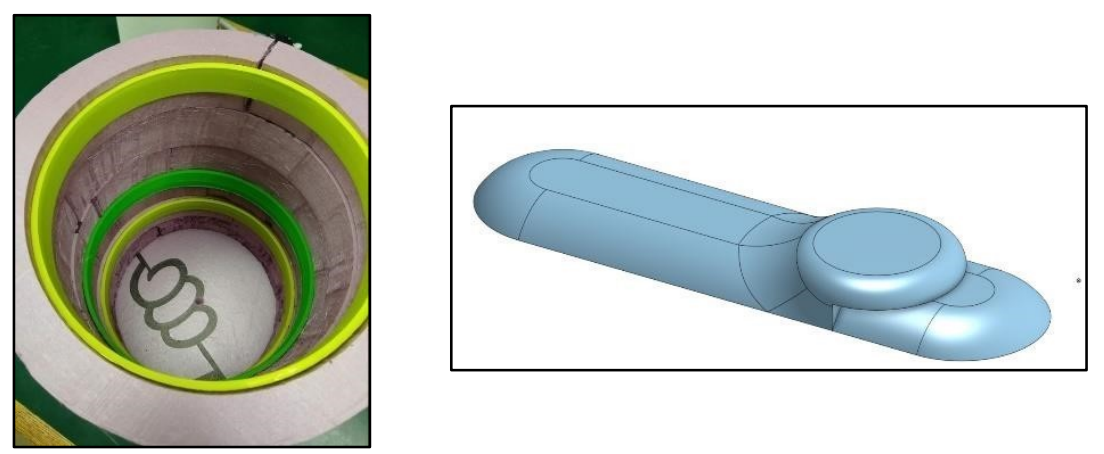

Figure 2. 3D Printed Guide Rings (left); "Snap and Lock" Model (right)

in Figure 2 depicts a small tab that is used to cause an interference fit between the inner and outer housing of the payload.

1.2 Methods Taking inspiration for the shelf design from 2017 Payload Team (Hennigan, Oliphant, Rasmussen, Rosenberger \& Vollmer, 2017), a 3D printed shelf along with laser cut bays were designed (Figure 3 ) The guide rings were 3D printed.

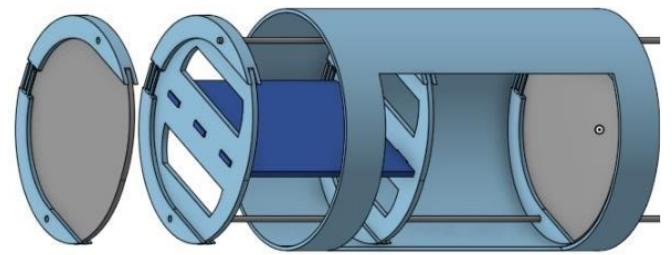

Figure 3. Payload Assembly

A small internal camera with internal LED lighting were installed into the electronics bay for internal recording of experiments. Additionally, an external 360-degree GoPro was mounted to the top of the payload for external recording. Images from both cameras can be seen in Figures 4 \& 5 .

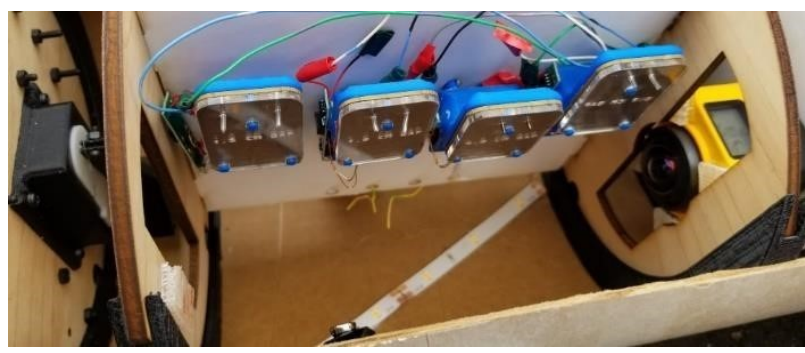

Figure 4. Internal Camera

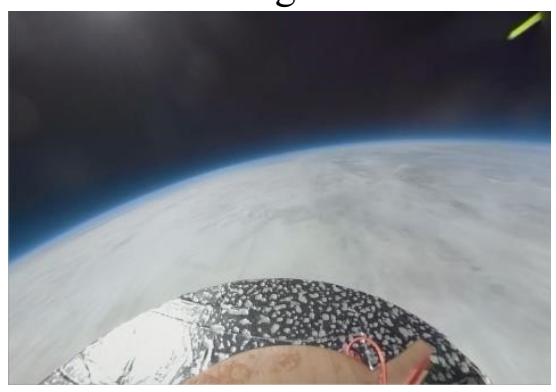

Figure 5. 360 Camera at $105,000 \mathrm{ft}$

1.3 Results Due to the time constraint, the, "snap and lock," model could not be integrated into the payload design for flight use. However, the guide rings proved to support the structure of the rocket tube well. The payload remained entirely intact through ascent and descent. There was no physical evidence to conclude that there was any disruption in the structure itself to elicit faulty data.

1.4 Conclusion The guide rings for the outer payload casing proved extremely effective. The low friction interaction between the PLA plastic and rocket body tube reduced assembly time on the field. Future design teams should consider increasing the number of guide rings or creating an entire 
PLA plastic sleeve to cover the inside of the payload shell. One issue experienced was due to the precision tolerance of the guide rings. The rocket body tube had to be inserted concentrically and parallel to the mating surface for smooth assembly. Any deviation in angle or tilt from the user would cause it to catch on a lower guide ring.

\section{Power Generation}

The objective of the power generation experiment was to generate electrical power output from the turbulence the high-altitude payload would experience in flight and use it to charge a battery.

2.1 Introduction With the increasing demand for renewable energy sources and following the project guidelines, the search for clean energy led the team to the skies. According to Ken Biba, as a high-altitude balloon ascends to $100,000 \mathrm{ft}$, its kinematics are dramatically affected by changes in wind speed, which varies from $20 \mathrm{mph}$ to $60 \mathrm{mph}$ throughout the flight (Biba, 2009). This experiment took advantage of the payload's naturally chaotic motion to create an electrical output. Capturing energy from an external source of generation, or energy harvesting, through this process has many practical applications, including the potential to supply power to remote electronic devices in weather balloons.

2.2 Methods The design constraints included physical size, electrical power output, reliability, and mass. Designs considered included: a counterweight mechanism, magnet-coil device from the 2017 payload team (Hennigan, Oliphant, Rasmussen, Rosenberger \& Vollmer, 2017), and a wind turbine. The design constraints as well as the research conducted converged towards the counterweight mechanism shown in Figure 6.

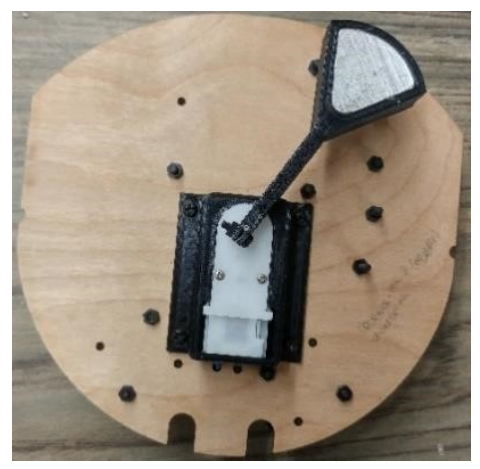

Figure 6: Counterweight Mechanism

A motor was asymmetrically loaded with a counterweight. As the payload was to experience turbulence, the counterweight would to spin the motor, generating electrical power. Initial testing consisted of attaching fishing weights to different motors and oscillating the motors while reading the voltage produced with an oscilloscope. This simple test showed the feasibility of the design concept while allowing for the optimization of the mass of the counterweight and the moment arm for each motor. Depending on the gear ratio of the motor used, this mechanism generated voltages between $0.1 \mathrm{~V}-3 \mathrm{~V}$. Based on the design constraints, previous years' flight footage, and inferences made from testing, a 120:1 motor was selected for final testing, and the most effective shape of the counterweight was determined.

To maximize efficiency while adhering to size and mass requirements, a $64.6 \mathrm{~g}$ counterweight was cut out of steel using a CNC machine and $48.5 \mathrm{~mm}$ counterweight holder was 3D-printed out of PLA plastic. A motor holder was 3D-printed out of PLA plastic, allowing for secure mounting of the counterweight mechanism to the wooden payload shelf - an aspect critical for ensuring maximum energy harvesting. All necessary electronics were mounted to the underside of the shelf. 


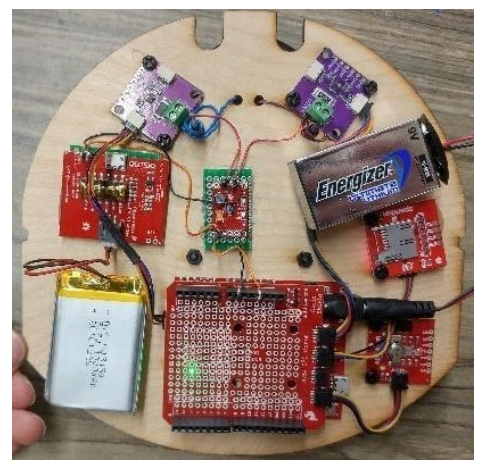

Figure 7. Power Generation Circuit
Figure 7 shows the circuit used to capture energy and measure it. The circuit included two voltage sensors for recording the voltage generated from both clockwise and counter-clockwise rotations of the counterweight, a four-bridge rectifier to convert the alternating current into direct current, and a capacitor to ensure an even flow of current to the lithium-polymer battery charger. Data from the voltage sensors was synchronized with a real-time clock and recorded on a micro-SD card. Before flight, the lithium-polymer battery was fully discharged; the time it took to deplete the battery was recorded for

comparison to the voltage sensor data post-flight.

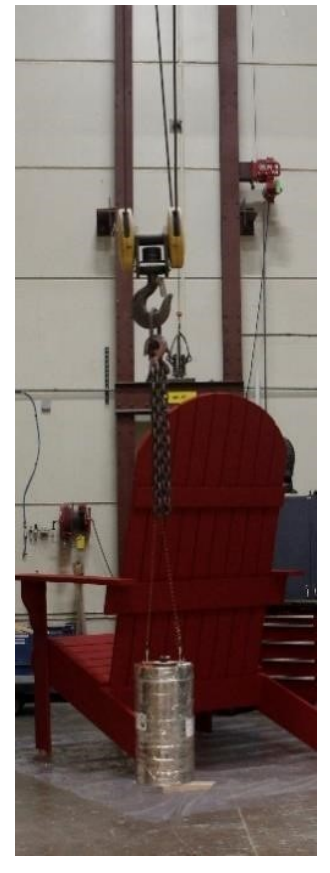

Figure 8: 2-Axis Crane Experiment
2.3 Results No flight data was captured due a structural failure in the payload, which snapped the counterweight holder, and electrical failures shortly after launch. To analyze the experiment's theoretical performance, two postflight tests were performed to simulate the motion experienced in flight with different levels of turbulence, according to the captured payload video. The first test was conducted by exciting the payload manually, and the second test was conducted by suspending the payload from a two-axis crane and exciting it remotely, as shown in Figure 8. The total energy produced was calculated by multiplying the known voltage and current values by the time it took to discharge the battery after testing, as shown in Eq. 1.

$$
E=\text { VIt }
$$

The total energy accumulated from the first test was $120 \mathrm{~J}$, and the energy accumulated in the second test was $96 \mathrm{~J}$. The values produced from these testing conditions suggest that with longer exposure to the more intense levels of turbulence in flight, the counterweight mechanism could produce enough energy to power electronic devices, such as an Arduino, for the duration of the flight. (“Arduino Uno Rev 3 Technical Specifications", n. d.)

\subsection{Conclusion}

No true conclusion can be drawn due to structural and electrical failures in flight. However, despite the small sample, the data collected from flight and testing indicates that the counterweight mechanism would be an effective energy harvester, especially during more severe levels of turbulence. The data also indicates that this system has the potential to power electronic devices throughout the flight. Further experimental trials would be conducted to reach more conclusive results. To avoid counterweight fracturing and electronic failures and described in Section 2.3, future flights should incorporate a counterweight holder 3D-printed from ABS plastic instead of the more brittle PLA (Kuentz, Salem A., Singh, Halbig \& Salem J. A., 2016) and ensure the functionality of all electronic components and proper data recording. 


\section{Turbulence}

Turbulence is mainly thought of in accordance to plane travel, but turbulent flow occurs whenever there is excess kinetic energy in a fluid flow that overcomes the damping effect of the fluid's viscosity. (Wyngaard, J., 1992) In other words, the flow velocity of the fluid is chaotic and unpredictable, which is why many physicists have deemed turbulence one of the greatest unsolved mysteries in physics. For the scope of this project, the overall goal was aimed at getting a better understanding of turbulence experienced by airliners. By tracking the kinetic motion of the payload and looking at energy dissipation rates (Weinstock, J., 1980), we can understand the turbulence experienced at different points during flight. This grants us the ability to find patterns with conjunction to environmental factors (temperature, pressure, etc.), and by using 3D animation software, one is able to visualize these relationships.

3.1 Methods Utilizing an IMU sensor, which includes an accelerometer (measures acceleration data), gyroscope (measures rotational data), and magnetometer (measures magnetic force), in combination with a GPS (measures the location or position) and an environmental monitor that collects data for temperature, pressure, and humidity, meaningful relationships with turbulence could be made.

Early in the design process, obtaining positional accuracy from IMU data was determined as a critical concern. Integrating acceleration twice to get position also integrates the error twice, which gives rise to error propagation. Additionally, the stand-a-lone GPS sensor produced low positional accuracy. To obtain accurate measurements of the position, a Kalman filter was used. The Kalman filter that was developed combined the measurements from the accelerometer and GPS, including their errors, to produce estimates of the position of the payload during flight using matrices, as shown in Eq. 2.

$$
\left[\begin{array}{l}
p \\
v
\end{array}\right]=\left[\begin{array}{cc}
1 & \Delta t \\
0 & 1
\end{array}\right] * \text { Previous State }+\left[\begin{array}{c}
\frac{1}{2} \Delta t^{2} \\
\Delta t
\end{array}\right] a
$$

These predictions of positions tend to be more precise than positions gathered from the accelerometer or GPS alone. From there placement of the IMU's became important. Instead of having a singular IMU that tracks the data from a single point on the payload, we decided that having a backup would be wise, with the main location being the center of mass.

When combining the acceleration data with the GPS using the Kalman filter, and including the rotational data, the full kinetic motion of the payload was described. A python script was developed to animate the rotation of the payload in an open-sourced 3D animation software (Community, B. O., 2018). Due to technical difficulties experienced right before flight, the IMU and GPS failed to collect usable data, and so the animation that we rendered was from the 2017 flight (Hennigan, N. M. W., Oliphant, S., Rasmussen, T. T., Rosenberger, F. M., \& Vollmer, B. T., 2017). 
Connecting all of this to turbulence, the hope was to look at the total energy of the payload at a starting portion of the flight path and at an ending portion of the flight path, and that gives an estimate of the total dissipated energy. Then by looking at the fluctuations in velocity during flight, we can better understand the turbulence experienced. Taking this a step further, we can compare the turbulence experienced at different altitudes, temperatures, pressure, magnetic forces, and humidity.

3.2 Result Currently, the typical way that meteorologists measure turbulence is by determining atmospheric stability. This is essentially the atmosphere resisting vertical motion. This is accomplished by taking vertical measurements of humidity and temperature compared to altitude (Figure 9). This sample data is then compared to a control at sea level. With these data samples, it is possible to assign a turbulence stability score given by how volatile humidity and temperature fluctuate. ("Turbulence Background, II." $E A I$ )

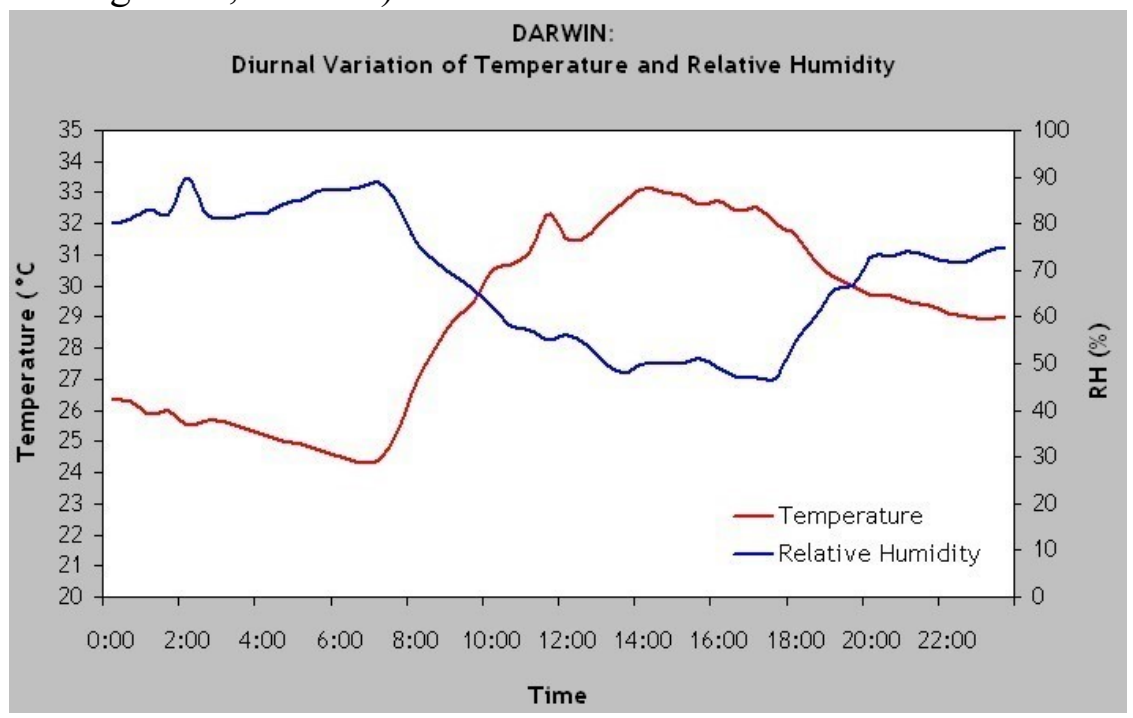

Figure 9. Plot of Temperature and Humidity Over Time for fixed altitude

Although this provides a general understanding into turbulence, a Lagrangian perspective could aid higher fidelity descriptions of turbulence. By capturing the acceleration of the balloon, it behaves like a fluid particle to precisely capture the turbulent behavior. By combining a Lagrangian prospective with a general assessment of atmospheric stability, a deeper insight into turbulence could be provided. To visualize this exact motion, a simple program in Python read in a CSV file containing similar atmospheric data with the addition of the kinetic motion of the payload. The program then subsequently created graphs of acceleration, angular velocity, temperature, humidity, etc. vs. time. Then, adding this into the Blender program, we have a visualization of this data. This should aid in the understanding of Figure 9, because a visual movement of the payload in a layer of air is easier to detect than by looking at the graph. Looking to the future, comparing these methods fully would be ideal. 


\section{Electronics Introduction}

The electronics experiments of the payload were designed with the intent of examining the effects of high altitude on electronics themselves, particularly on printed circuit boards (PCBs). From the (Blattau, N., \& Hillman, C. (n.d.)), there is a notable change to electronics reliability at high altitudes. This paper only explored conditions at altitudes up to 40,000 feet. Additionally, (Schweickart, Daniel, John, et al. (n.d.)) brings up the design considerations for PCBs when used with high voltages at high altitudes. This led to the experimental idea for the ionization energy of air. Beyond the ionization energy of air other considerations are required when designing electrical components for use in high altitude devices such as the impact of increasing radiation at altitude.

4.1 Ionization Energy of Air As the density of air changes the amount or air required to ionize, or breakdown the air changes non-linearly. This is related to how the resistivity of air changes with density. Ionization energy is the amount of energy required to break electrons free from the parent atom and cause them to align in what we see as an arc. This relationship between ionization energy and pressure can be expressed by a Paschen Curve (Isidorovic, J, 1995). This voltage behavior can be seen when plotted against pressure in Figure 10.

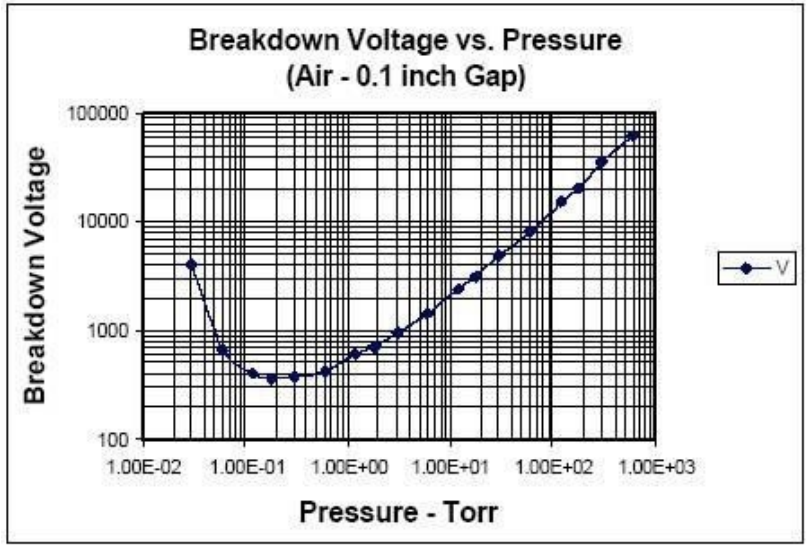

Figure 10. Example of Breakdown Voltage vs Pressure (highvoltageconnection.com)

To examine the changes in ionization energy of air, several off the shelf arc-lighters (Figure 11)

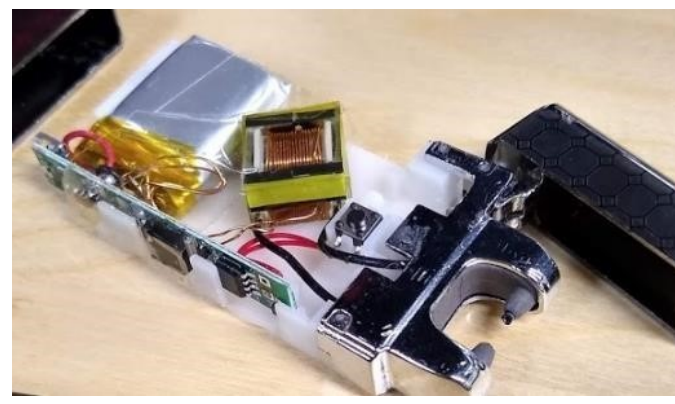

Figure 11. Disassembled Arc Lighter

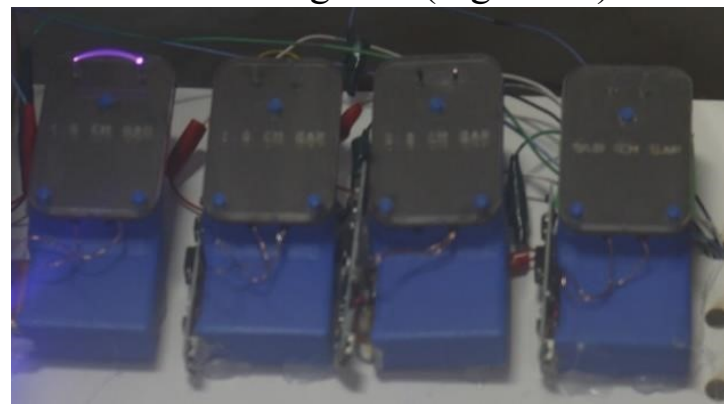

Figure 12. Ionization Apparatus

were purchased and disassembled. The disassembled lighters were then placed into a 3D printed holder. A lid for the 3D printed holder was designed and cut on a laser cutter to precisely space the arc gaps at predetermined distances (Figure 12). The gaps were set to distances of $1.5 \mathrm{~cm}, 1.0 \mathrm{~cm}$, $0.8 \mathrm{~cm}, \& 0.5 \mathrm{~cm}$ respectively. 
The gap distances were determined based on arcing characteristics at atmospheric pressure. In theory, $0.5 \mathrm{~cm}$ and $0.8 \mathrm{~cm}$ gaps would readily arc at atmospheric pressure given the transformer voltage and comparing ionization requirements with Paschen curves, the $1.0 \mathrm{~cm}$ gap would intermittently arc and the $1.5 \mathrm{~cm}$ gap would not arc.

As the altitude increased, we expected to see the $1.0 \mathrm{~cm}$ gap begin to arc readily. As the air pressure begin to decrease due to elevation change, the $1.5 \mathrm{~cm}$ gap arced as the air became even thinner. Through lab testing in a vacuum chamber this phenomenon was seen clearly, as expected. An unexpected result occurred when the air pressure was decreased further. The girth of the arcs continually increased until a thick band of ionized air was seen (Figure 13).
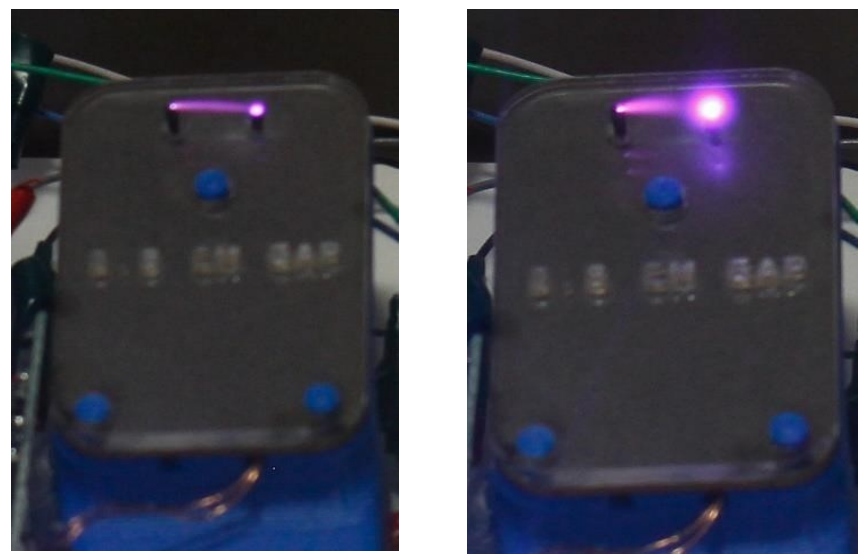

Figure 13. Ionization of the $1.5 \mathrm{~cm}$ Gap in a Vacuum

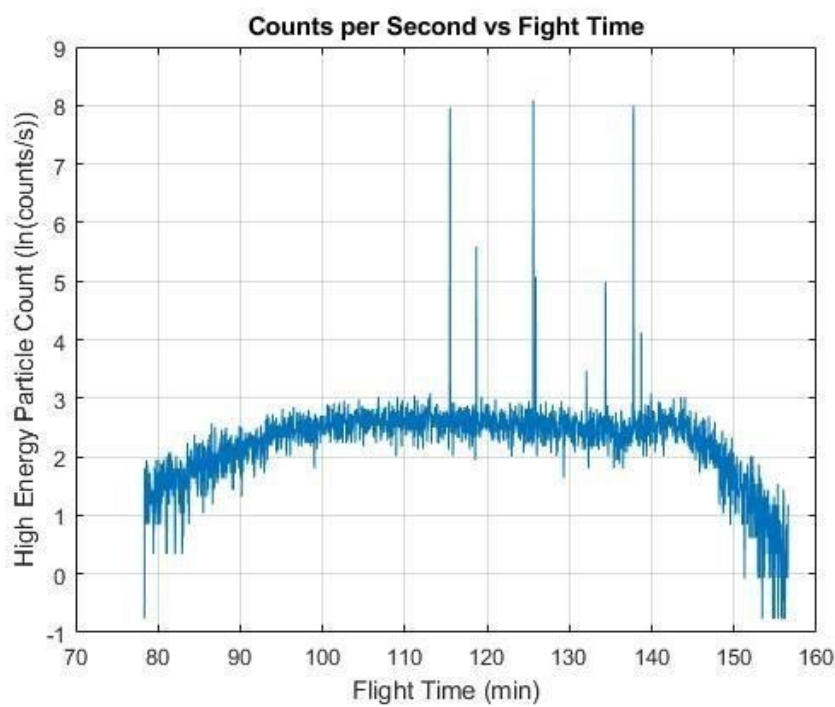

4.2 Changes in Radiation with Altitude

The primary way which radiation affects electronics is by introducing unexpected amounts of energy into the circuit. The extraneous energy can then cause errors as electricity is introduced into the circuit randomly. This random energy can cause erroneous data values and potentially corruption on storage devices. To measure changes in radiation, a Geiger counter was placed in the payload, on one of the upper shelves near the outer edge to maximize the exposure to radiation. The selected Geiger tube was a Figure 14: Plot of the Number of High-Energy Particles Over Flight Time halogen tube sensitive to gamma and beta radiation. The predicted changes in detectable gamma and beta rays are shown alongside the actual flight in Figure 14. Gama and beta radiation were aggregated into "High Energy Particles" and counted for the duration of the flight. Throughout the flight the amount of radiation detected increased steadily as expected and came to level off near 12 counts per second at peak. Beyond the peak there are several spikes of where the counts per second exceed 2,500. 
4.3 Conclusion Paschen curve behavior was demonstrated in laboratory vacuum experiments and correlated well with published results (Isidorovic, J, 1995). The unique corona effect shown in Figure 13, demonstrated the ability for low density air to readily ionize. When considering high voltage experimentation at upper atmosphere, this ionization characteristic should be considered when designing experiments or apparatuses for low earth-orbit/high-altitude sciences. The difference in insulation design criteria between sea level and potential operating conditions are so drastic, conservative measures should be implemented when designing circuitry for open air operation. Additionally, radiation detection observed an increase in high energy particles during flight as the balloon reached higher atmosphere conditions.

\section{Sky Music - Sonification of Flight Data}

\section{Introduction}

When sonifying data in a direct data-to-frequency relationship, one must balance data resolution with musical tonality. Higher resolution increases the presence of atonal or microtonal notes leading to complex and dissonant sounds. However, aiming for more westernized tonalities required filtering and truncating data to fit specific frequencies. In order to perceive data relationships through music, this balance became important. The Sky Music Project set out to explore new and innovative ways to perceive and analyze data using sonification while maintaining as much of the nuance in the original data. Using musical elements such as harmony and pitch, the team was able to perceptualize data through music. All code and media examples can be found at (https://wsgcballoonpayload.wixsite.com/2019team)

5.1 Data Processing Post-processing of the data gathered during experimentation and flight was handled in Python. Python Programming Language was chosen due to its robust, dataanalysis libraries: NumPy, Pandas, and Matplotlib. All three of these libraries were used to organize and process data from the CSV file generated from the payload's SD cards. These libraries were then used in conjunction to organize data into a more efficient structure for analysis. The Pandas Library, which is built upon the NumPy Library, organized data into Data Frames. The Pandas Library offers numerous objects for data frames ideal for indexing/referencing data. Efficiently organized data allowed for simple graphical plotting to spot initial trends before sonification.

5.2 Sound Synthesis The sonification of data program was written in ChucK. ChucK is an open-source programming language for real-time sound synthesis and music creation (Kapur, 2015). ChucK contains several classes and objects that allow for precise time, pitch, and stereoplacement manipulation. Importing the requisite data streams from Python-generated CSV files, ChucK used the data to drive musical elements such as stereo placement, or pitch, and even harmonic progressions in more complex applications.

5.3 Method The sonification program was influenced by the works of Brian Foo. Specifically, his project titled "Two Trains". In this project, Mr. Foo sought out to communicate the differences in median household income in NYC neighborhoods along the path of famous 2Train. His implementation of Python and ChucK Programming Languages were direct inspiration for the programs we developed. Much like Mr. Foo, our data read from SD Card File to Python and 
organized into easy-to-use data frames. Temperature (Figure 15) and Pressure (Figure 16) data gathered by the 2016 Elijah Balloon Project for the development phase.

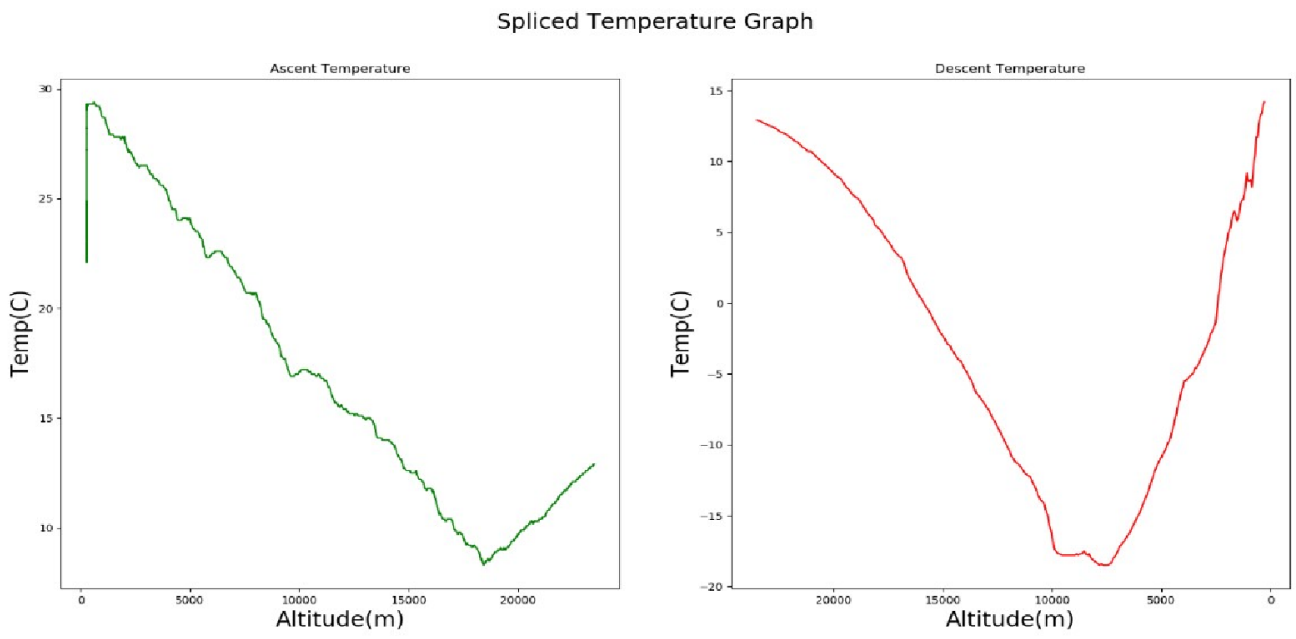

Figure 15. 2016 Elijah Balloon Project Pressure Data
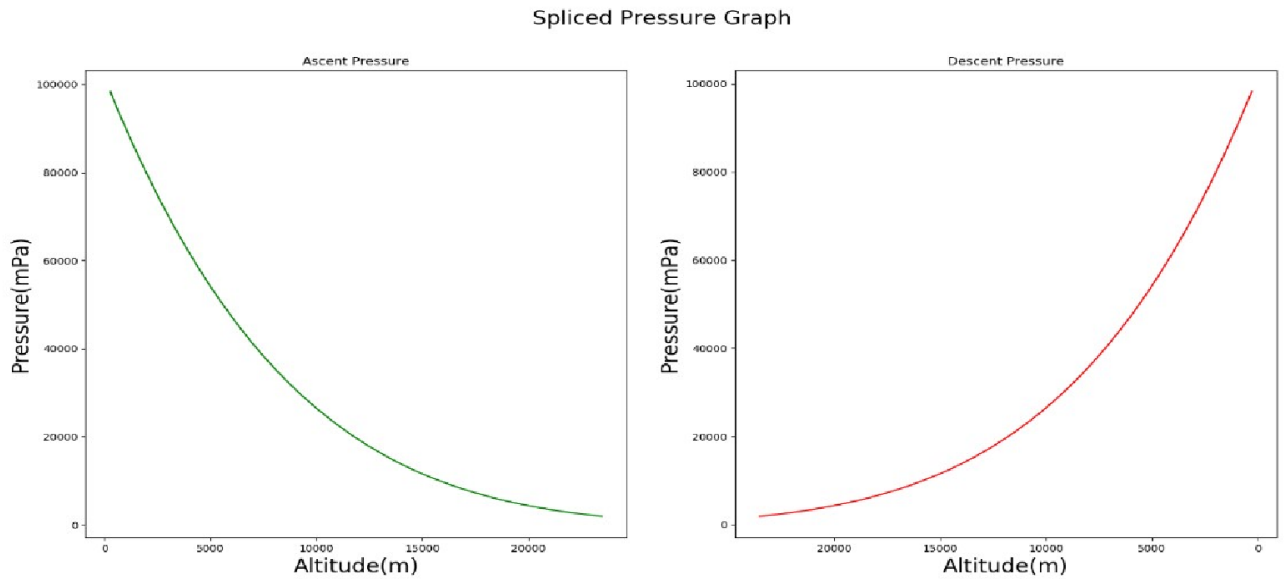

Figure 16. 2016 Elijah Balloon Project Temperature Data

Data was then processed through basic statistical and graphical analysis methods and written to files for ChucK to read. From this, two experiments were created. In the first, temperature data was used to determine the stereo placement of a single pitch, with right representing positive temperatures and left representing negative. A balanced left-right audio signal represented 0 on the number line. In the second experiment, pressure data was used in the conditional logic for decision-making, therefore determining a harmonic progression for the hardcoded musical composition. You'll find a general flowchart of how programming languages were implemented in Figure 17.
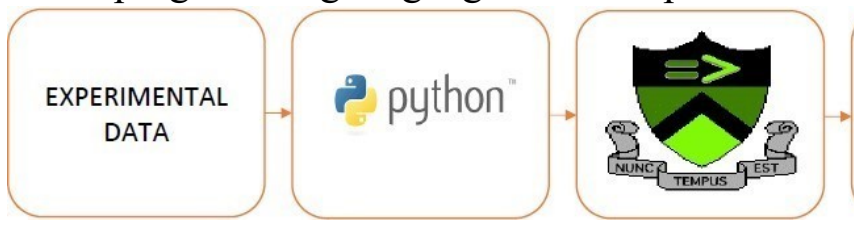

MUSIC

Figure 17. Flow of Programming Languages 
5.4 Conclusion Sonification of data was successful in allowing users to perceive data trends through the music while maintaining musical composition. However, building more complex compositions proved challenging. The manipulation of global variables in ChucK is not supported very well. Additionally, the language has very little documentation on the process. The discrepancy between the ambiguously defined global and local spaces renders the final program incomplete with respect to the vision of a rich composition. While the script runs, the inability for variable assignments to be maintained throughout the iterations of looping breaks the conditional logic setup for the pressure data in determining the harmonic progression. Moving forward, we would implement the 3-function workaround for global variables in ChucK. In the initial brainstorming of the musical structure of the algorithm, the team had to analyze sample data to assure the generated music abided by western tonal harmony. In developing the skeleton for the algorithm, traditional data analysis methods were used to develop the structure for the program. In retrospect, this was deemed counterintuitive to the goals of our research. With this in mind, we considered how the execution of the Sky Music Project might differ with this approach: trying to discover the data in the music rather than the music in the data.

\section{Acknowledgments}

Dr. William Farrow

Vivian Mickelson

\section{References}

Arduino Uno Rev 3 Technical Specifications (n. d.). Retrieved from https://store.arduino.cc/usa/arduinounorev3

Breakdown Voltage: Paschen Curve: Altitude and Pressure: Corona: High Voltage Spacing: Insulation: from High Voltage Connection. (n.d.). Retrieved from http://www.highvoltageconnection.com/articles/pasche n -curve.html.

Biba, K. (2009). High Altitude Wind Speed, Direction, and Air Temperature at Black Rock Nevada for Amateur Rocketry Application

Blattau, N., \& Hillman, C. (n.d.). Failure Mechanisms in Electrical Products at High Altitudes. Retrieved from https://pdfs.semanticscholar.org/6046/529f05a65e182 $\underline{\mathrm{a}}$

\section{3ef8eb26b2075d0e13.pdf}

Community, B. O. (2018). Blender - a 3D modelling and rendering package. Stichting Blender Foundation, Amsterdam. Retrieved from http://www.blender.org Foo, B. (n.d.). Data-Driven DJ. Retrieved from https://datadrivendj.com/
Justin Sommer

Brian Foo

Hennigan, N. M. W., Oliphant, S., Rasmussen, T. T., Rosenberger, F. M., \& Vollmer, B. T. (2017). 2017 WSGC Elijah High-Altitude Balloon Payload. New Windows: Gravitational Wave Astronomy. doi: https://doi.org/10.17307/wsc.v1i1.219

Home: WSGC 2019 Elijah High-Altitude Balloon Fellowship. (n.d.). Retrieved from https:/wsgcballoonpayload.wixsite.com/2019team

Isidorovic, J. (1995). PASCHEN CURVES AND SPATIAL DISTRIBUTION OF EMITTED LIGHT OF GLOW DISCHARGE IN THE AIR. (Acta Phys. Pol. A 88 Supplement, S-37 (1995).

Kapur, A., Perry, C., Salazar, S., Wang, G. (2015). Programming for Musicians and Digital Artists

Kuentz, L., Salem, A., Singh, M., Halbig, M. C., \& Salem, J. A. (2016). NASA Technical Reports Server. Retrieved from https://ntrs.nasa.gov/search.jsp?R=20160010284

"Turbulence Background, II." EAI, www.experimentalaircraft.info/flightplanning/turbulenc e-background-1.php. 
https://apps.dtic.mil/docs/citations/ADA522066

Weinstock, J. (1980). Energy Dissipation Rates of

Turbulence in the Stable Free Atmosphere.

Wyngaard, J. (1992). Atmospheric Turbulence

Schweickart, Daniel, John, Lawrence, Hatfield, Lynn,

... Hermann. (n.d.). A Guide to Electrical Insulation

Design in Aerospace Vehicles for a Broad Range of

Environmental Parameter Space. Retrieved from 